\title{
Feminismo de Estado en la Argentina democrática (1983-2021): ¿modelo aspiracional o realidad institucional?*
}

State Feminism in Democratic Argentina (1983-2021):

Aspirational Model or Institutional Reality?

\author{
DÉBORA LOPREITE**
}

Universidad de Buenos Aires, Argentina

deboralopreite@hotmail.com

\author{
ANA LAURA RODRÍGUEZ GUSTÁ ${ }^{1}$ \\ Universidad Nacional de San Martín, Argentina \\ alrgusta@unsam.edu.ar
}

https://doi.org/10.46468/rsaap.15.2.A2

\begin{abstract}
Resumen: Desde 1983 con la vuelta a la democracia hasta 2021, la agencia de política para las mujeres en Argentina muestra una trayectoria institucional no lineal. Utilizando una tipología de agencias propuesta por la teoría de Feminismo de Estado, este artículo caracteriza, en primer lugar, las diferentes expresiones institucionales de las agencias de políticas para las mujeres según su nexo con los movimientos de mujeres y feministas. En segundo lugar, el artículo explica estas variaciones a partir de las dinámicas y los cambios de los movimientos de mujeres y feministas, y el uso instrumental de las agencias de las mujeres, por parte del Poder Ejecutivo, en la negociación y regulación de conflictos con diversos actores. El artículo busca contribuir a los debates de feminismo de Estado enfatizando la heterogeneidad y dinámica de los movimientos feministas y las decisiones pragmáticas de los líderes políticos.
\end{abstract}

Palabras Clave: Agencias de políticas de las mujeres - Institucionalidad de género - Feminismo de Estado - Movimiento feminista - Argentina

\begin{abstract}
With the return of democracy in 1983 until 2021, the women's policy agency in Argentina shows a non-linear institutional trajectory. Using a typology of agencies proposed by the theory of State Feminism, this article characterizes, in the first place, the different institutional expressions of women's policy agencies according to their nexus with the women's and feminist movements. Secondly, the article explains these variations based on the dynamics and changes of women's and feminist movements, as well as the instrumental use of women's agencies by the Executive Power when regulating conflicts with conservative actors. The article seeks to contribute to the debates on State Feminism by emphasizing the heterogeneity of feminist movements vis-à-vis political leaders' pragmatic decisions at critical conjunctures.
\end{abstract}

Keywords: Women's policy agencies - Gender Institutions - State Feminism - Feminist Movement Argentina

* Artículo recibido el 3 de agosto de 2021 y aceptado para su publicación el 30 de septiembre de 2021.

** Las autoras agradecen el aporte de dos evaluaciones anónimas a una versión previa de este artículo.

$1 \quad$ La autoría está ordenada alfabéticamente. 


\section{Introducción}

En América Latina, las desigualdades y la discriminación por razones de sexo y género son problemas públicos que ganaron un lugar en la agenda a la luz de los procesos de democratización. La creación de la institucionalidad de género en el Estado ha sido una respuesta a este asunto, como resultado de la presión de movimientos de mujeres y feministas (Guzmán, 2001). Las agencias de políticas para las mujeres-conocidas como oficinas de la mujer, mecanismos para el adelanto de las mujeres o incluso maquinarias de género- son las estructuras estatales, de diferente jerarquía, con el mandato normativo de promover la mejora en la posición social de la mujer y/o la igualdad de género (McBride y Mazur, 1995).

La Tercera Conferencia Mundial de la Mujer en Nairobi (1985) fortaleció la agenda de la institucionalidad de género en el Estado. La conferencia concluyó con las "Estrategias de Nairobi para el Avance de la Mujer" que instaron a consolidar las agencias especializadas e introducir planes, programas y medidas legislativas que aseguraran la participación de las mujeres en distintos ámbitos (Çağatay, Grown, y Santiago, 1986). En 1995, la Cuarta Conferencia Mundial de la Mujer en Beijing mostró que estas agencias tenían mandatos confusos y carecían de suficientes recursos y apoyo político por parte de las autoridades nacionales. Como forma de avanzar, la Plataforma de Acción resultante instó a fortalecerlas y a adoptar el mainstreaming de género, es decir, una estrategia de políticas de largo plazo que incorporase la perspectiva de igualdad de género en la totalidad de las acciones estatales (Molyneux y Razavi, 2005). A más de 25 años, investigaciones regionales (Díaz García, 2016; Rodríguez Gustá, Madera y Caminotti, 2017) y estudios comparados recientes (Rodríguez Gustá, 2021) mostraron que las agencias de políticas para las mujeres continúan con debilidades en su institucionalidad y capacidades para la labor transversal vis-a-vis la creación de espacios consultivos con las organizaciones de mujeres, con excepciones muy específicas.

Este trabajo analiza los cambios institucionales de la agencia nacional para las mujeres en Argentina, desde el retorno de la democracia en 1983 al presente. Para la caracterización de la transformación institucional, resulta relevante recurrir al concepto de feminismo de Estado o agencia Insider (McBride y Mazur, 1995; McBride y Mazur, 2010) como forma institucional aspiracional. El abordaje histórico permite observar el proceso de construcción de estatidad (Oszlak, 1982), entendido como la capacidad del Estado de absorber las demandas del movimiento de mujeres, mediante estructuras estatales jerarquizadas y recursos para abordar los problemas plantea- 
dos. Al colocar el foco en la robustez institucional de las agencias para las mujeres, consideramos la capacidad estatal y la conformación de estructuras de autoridad estatal como consecuencias de las disputas por delimitar los problemas públicos merecedores de atención (Oszlak y O’Donnell, 1995). Para nuestro caso, la movilización feminista alrededor de las cuestiones de género y la perspectiva sustantiva o instrumental del Poder Ejecutivo de los asuntos de igualdad de género son centrales para comprender la calidad institucional de las agencias para las mujeres.

La estrategia analítica consiste en un abordaje longitudinal cualitativo para capturar las distintas configuraciones institucionales de la agencia de políticas de género en la Argentina post dictadura. Algunos estudios han caracterizado las agencias de políticas para las mujeres con el kirchnerismo y el gobierno de Cambiemos en perspectiva comparada (Rodríguez Gustá, 2021), pero el aporte original de esta investigación es la mirada histórica que permite examinar un amplio rango de variación institucional que de otra forma queda trunco por limitarse a gobiernos específicos. Esta mirada diacrónica contribuye a profundizar la heterogeneidad causal, al identificar cómo las transformaciones de los movimientos de mujeres y de las estrategias del Poder Ejecutivo incidieron en la configuración de la institucionalidad de las agencias. La tipología de la teoría de feminismo de Estado respecto de la calidad de los nexos entre la agencia y los movimientos de mujeres resulta útil para caracterizar las configuraciones institucionales y sus transformaciones. Las fuentes de información son documentos oficiales, estudios previos y entrevistas realizadas por las autoras ${ }^{2}$.

El artículo se organiza de la siguiente manera. Se plantea una tipología de agencias derivada de la perspectiva teórica de feminismo de Estado. Luego se examinan las sucesivas agencias de políticas para las mujeres en Argentina, desde la democratización iniciada en 1983 hasta nuestros días para analizar las diferentes configuraciones. Seguidamente, se plantean las interpretaciones respecto de los cambios en la configuración institucional, con énfasis en la dinámica de los movimientos de mujeres y el comportamiento del Poder Ejecutivo a la luz de coyunturas sociopolíticas críticas. Finalmente se presentan las conclusiones.

2 Se examinaron los siete informes de país presentados ante el Comité de la CEDAW entre 1984 y 2016 (último año de reporte), los decretos de creación y modificación de la institucionalidad de género, y los documentos oficiales de planes y programas. Asimismo, se utilizaron entrevistas realizadas en dos momentos: cinco entrevistas de 2005 a 2008 y cinco entrevistas de 2018 y 2019. Fueron entrevistas a autoridades de la agencia, así como también representantes del movimiento social. 


\section{Feminismo, movimiento de mujeres y agencias de políticas para las mujeres}

En Europa y América del Norte se generó una corriente de investigación denominada Feminismo de Estado (state feminism) con dos estudios seminales: Comparative State Feminism (1995) y The Politics of State Feminism (2010). A partir de la Red de Investigación en Política de Género y Estado (RNGS, por su sigla en inglés), estos estudios abordaron cómo y en qué condiciones las estructuras del Estado adoptaban perspectivas de igualdad de género y políticas feministas, en vinculación con el movimiento de mujeres. Se buscó comprender la consistencia y la variación de las agencias de políticas para las mujeres en tanto entidades mediadoras entre los movimientos de mujeres y el Estado. El núcleo central de análisis es el nexo entre la agencia de políticas para las mujeres y los movimientos con aspiraciones feministas (McBride \& Mazur, 2010), es decir, la interrelación entre las autoridades de la agencia y el movimiento de mujeres y feministas en diversas arenas sociales y políticas. Esto puso el foco en cómo las agencias posibilitan el acceso y la influencia de los movimientos a las decisiones de política. La clave reside en la relación y articulación entre las agencias y los movimientos, como condición sine qua non para consolidar una política pública feminista.

Desde este ángulo, McBride y Mazur $(2010,18)$, con base en democracias post industriales, ofrecen una tipología de agencias de políticas para las mujeres: agencias Insiders (aliadas), marginales, simbólicas y antifeministas. Las agencias Insiders son aquellas que facilitan los debates políticos enmarcados en los términos de las demandas de los movimientos de mujeres y, en consecuencia, introducen la justicia y la igualdad de género en las normas y regulaciones. Estas agencias son las que las autoras consideran una expresión del Feminismo de Estado ya que lograrían políticas públicas feministas, gracias a las estructuras de consulta y diálogo con el movimiento de mujeres. Sin embargo, las agencias pueden ser marginales cuando carecen de las capacidades para promover los derechos de las mujeres y las políticas de igualdad de género, por lo que tienen dificultades al momento de redistribuir el poder social (McBride y Mazur, 2010). Las agencias son simbólicas cuando están excluidas de los principales debates políticos y sin vínculos con los movimientos feministas. Así las cosas, estas agencias quedan al margen de las demandas de los movimientos y de la agenda política. Las autoras consideran la posibilidad de que existan agencias antifeministas cuando las políticas se sustentan en ideas contrarias a los derechos humanos de las mujeres y los nexos son conflictivos y expresan relaciones antagónicas con el movimiento de mujeres. 
El punto de mayor tensión causal es la noción de generización (gendering) entendida como "cuándo y bajo qué circunstancias las agencias estatales son aliadas con los actores del movimiento feminista para desafiar las bases patriarcales del propio Estado" (McBride y Mazur, 2010: 24). Aquí es donde la calidad del nexo entre las agencias y el movimiento se torna central a fin de determinar en qué grado estas efectivamente logran instalar políticas públicas beneficiosas para las mujeres (Kantola y Outshroom, 2007) y ser Insiders. Solamente en estas condiciones, las agencias de políticas de mujeres han sido efectivas en incluir una perspectiva feminista en los debates públicos y proporcionar acceso al proceso de toma de decisiones a los actores sociales, democratizando la agenda pública (Mazur, 2012).

En América Latina, Rodríguez Gustá et al. (2017) mostraron que gran parte de las agencias para las mujeres presentan un carácter híbrido, por las debilidades de los nexos con los grupos de mujeres y feministas. Experiencias tempranas como el Servicio Nacional de la Mujer (SERNAM) tuvieron conflictos en su interacción con segmentos del movimiento (Franceschet, 2003), lo cual complicó su papel de agencia Insider especialmente para mujeres indígenas (Richards, 2004). Una notoria expresión del feminismo de Estado fue, en Brasil, la Secretaría Presidencial de la Mujer bajo el Gobierno del Partido de los Trabajadores (Bohn, 2010). Ahora bien, el actual gobierno de Jair Bolsonaro la transformó en una agencia antifeminista por su conservadurismo social y relación antagónica con el movimiento feminista (Rodríguez Gustá, 2021). Otros abordajes señalaron las dificultades de las agencias en adoptar un enfoque propiamente de derechos (Astelarra, 2004), o incluso de poder transmitir un marco de sentido transformador a los niveles subnacionales (Zaremberg et al., 2019).

Respecto de los factores que darían cuenta de la configuración institucional de las agencias, se destaca la participación y representación política de las mujeres, consideradas como sujetos políticos y centro del análisis, con énfasis en su capacidad política de incidencia en las agencias para las mujeres (Lovenduski, 2005). Al respecto, un estudio reciente de Zaremberg y Almeida (2021) sobre México y Brasil, señala cómo las feministas en cargos institucionales ofemócratas, establecen redes a lo largo de diversos segmentos del Estado que protegen de los embates conservadores. Así, el feminismo de Estado no se desarrollaría sin las mujeres en los partidos políticos y en las élites estatales (Eisenstein, 1996), por lo que estas redes serían un factor fundamentales de cuándo y cómo las agencias de las mujeres serían Insiders. 
Tempranamente, Hernes (1987) identificó que las estructuras favorables a las demandas feministas son un proceso "desde arriba" gracias a la presencia de profesionales feministas en las áreas relevantes del Estado, pero siempre acompañado de otro "desde abajo" con la movilización de mujeres en ámbitos culturales, sociales y políticos no institucionalizados (Hernes, 1987). Por lo tanto, una agencia Insider aparecería ligada a la acción política de las mujeres mediante su movilización social, así como a su representación institucional. Precisamente, en el caso de Brasil, la cualidad de feminismo de Estado implicó un nexo estrecho entre un activo y diverso movimiento de mujeres y la Secretaría de la Mujer, más el activismo de las feministas a lo largo del Estado (Bohn, 2010; Rodríguez Gustá, 2021).

Respecto del movimiento de mujeres, una de sus características es la multiplicidad, variedad y diversidad de organizaciones que lo conforman en un campo de acción diverso (Sutton, 2020). Más allá de que en ciertas coyunturas el movimiento se articula y arma redes para dar debates trascendentales, su heterogeneidad influye en la estrategia para conformar alianzas de largo plazo con las agencias estatales. En otras palabras, los diferentes segmentos del movimiento de mujeres y feministas inciden en cómo se desarrolla el nexo con las agencias de políticas para las mujeres. Esto puede variar según la radicalidad de los grupos, su adscripción política, la pertenencia de clase y estatus, e identidades étnico-raciales. En América Latina, una distinción conocida se dio entre feministas autónomas y feministas institucionalizadas que, hacia mediados de los noventa, distanció y enfrentó a buena parte del movimiento con la institucionalidad estatal de género (Álvarez, 2009). Esto no implicó una ruptura irreconciliable, pero sí una reconfiguración de los nexos, volviéndolos en ocasiones informales e indirectos. Recientemente, las feministas jóvenes, queer y performáticas, persiguen una estrategia "desestructurada" y estarían menos inclinadas a interactuar con las estructuras estatales, como lo ilustran Chile (Miranda y Roque López, 2019) y Argentina (Manzano, 2019). Sin embargo, es una realidad que en la región el feminismo se ha transformado en un movimiento multifacético, con una intensa presencia en los ámbitos sociales e institucionales y con nexos con el Estado (Levy y Bohn, 2021).

En tanto agencias del Poder Ejecutivo, la gestión e ideología gubernamental incide en la institucionalidad, en particular en contextos hiperpresidencialistas como los latinoamericanos. En Europa se identificó que un gobierno de izquierda es fundamental para algunos resultados políticos feministas, pero no todos (McBride y Mazur, 2010; Weldon, 2002). Las agencias son más efectivas solamente bajo aquellos gobiernos de la 
socialdemocracia que priorizaron las cuestiones de género en sus agendas (Noruega, Dinamarca, Australia y Holanda) (McBride y Mazur, 1995). En América Latina, las agencias de las mujeres son sensibles a las coyunturas políticas y económicas (Fernós, 2010), y no parecerían depender de lo establecido en plataformas programáticas o en los compromisos electorales (Rodríguez Gustá et al., 2017). En efecto, existiría una relación no lineal entre la ideología del Poder Ejecutivo y la consolidación de las estructuras de género en el Estado, incluso bajo gobiernos de centro izquierda o de la denominada "marea rosa" (Friedman y Tabbush, 2019). En realidad, investigaciones recientes identificaron que los temas de género son fácilmente negociables (Friedman y Tabbush, 2019) e incluso "moneda de cambio” en la transacción política (Rodríguez Gustá, 2021). Por ello, las agencias para las mujeres (y otros temas de igualdad de género) pueden adquirir valor instrumental para los líderes políticos ya que responden a intereses de distinta influencia política y su relevancia varía significativamente de acuerdo con el contexto.

\section{Cambios institucionales de la agencia de políticas para las mujeres en Argentina}

Siguiendo la tipología de Manzur and McBride Stetson (2010), esta sección aborda las configuraciones de las agencias de políticas para las mujeres, desde 1983 cuando nace como Subsecretaría de la Mujer hasta el actual Ministerio de las Mujeres, Géneros y Diversidad creado en 2019. El análisis considera las administraciones gubernamentales con ciclo completo, por la cual no se toman los gobiernos de la Alianza (De La Rúa, 19992001) ni de Eduardo Duhalde (Partido Justicialista, 2002-2003). Como se resume en la tabla 1, los cambios se manifiestan en tres dimensiones: (i) los marcos de sentido de las intervenciones, (ii) en las preferencias de las autoridades de las agencias y (iii) las estructuras consultivas formales disponibles. Respecto de los marcos de sentido, utilizamos la clasificación de Molyneux (1985) de intereses "prácticos" de género -referidos a la satisfacción de necesidades básicas de vida, la reproducción social de los hogares y las comunidades- e intereses "estratégicos" -de mayor alcance político porque apuntan al cuestionamiento de las relaciones de subordinación del orden de género y a su transformación (Molyneux, 1985: 233). 


\section{TABLA 1}

Evolución de la configuración institucional de la agencia de políticas para las mujeres, 1983-2021.

\begin{tabular}{|c|c|c|c|c|c|c|c|}
\hline DIMENSIÓN & $\begin{array}{l}\text { Subsecretaría } \\
\text { de la Mujer } \\
1987-1989\end{array}$ & $\begin{array}{l}\text { Consejo } \\
\text { Nacional de } \\
\text { la Mujer } \\
\text { (etapa 1) } \\
\text { 1992-1994 }\end{array}$ & $\begin{array}{l}\text { Consejo } \\
\text { Nacional de } \\
\text { la Mujer } \\
\text { (etapa 2) } \\
\text { 1995-1999 }\end{array}$ & $\begin{array}{l}\text { Consejo } \\
\text { Nacional } \\
\text { de la } \\
\text { Mujer } \\
\text { 2003- } \\
2007 \\
\end{array}$ & $\begin{array}{l}\text { Consejo } \\
\text { Nacional } \\
\text { de las } \\
\text { Mujeres } \\
\text { 2008-2015 }\end{array}$ & $\begin{array}{l}\text { Instituto } \\
\text { Nacional } \\
\text { de las } \\
\text { Mujeres } \\
\text { 2015-2019 }\end{array}$ & $\begin{array}{l}\text { Ministerio } \\
\text { de las } \\
\text { Mujeres, } \\
\text { Género y } \\
\text { Diversidad } \\
\text { 2019-2021 }\end{array}$ \\
\hline $\begin{array}{l}\text { Marcos de sentido } \\
\text { de las intervenciones } \\
\text { de la agencia }\end{array}$ & $\begin{array}{l}\text { Intereses } \\
\text { estratégicos }\end{array}$ & $\begin{array}{l}\text { Intereses } \\
\text { estratégicos } \\
\text { (participación } \\
\text { política, } \\
\text { empleo, } \\
\text { DSDR, } \\
\text { educación) } \\
\end{array}$ & $\begin{array}{l}\text { Interpretación } \\
\text { conservadora } \\
\text { de intereses } \\
\text { prácticos }\end{array}$ & $\begin{array}{l}\text { Intereses } \\
\text { prácticos } \\
\text { (empleo) }\end{array}$ & $\begin{array}{l}\text { Intereses } \\
\text { estratégicos } \\
\text { limitados a } \\
\text { violencia } \\
\text { de género }\end{array}$ & \begin{tabular}{|l} 
Intereses \\
estratégicos, \\
limitados a \\
violencia de \\
género
\end{tabular} & $\begin{array}{l}\text { Intereses } \\
\text { estratégicos }\end{array}$ \\
\hline $\begin{array}{l}\text { Preferencias de las } \\
\text { lideresas }\end{array}$ & Feminista & Feminista & $\begin{array}{l}\text { Anti abortista } \\
\text { católica }\end{array}$ & $\begin{array}{l}\text { Anti } \\
\text { abortista } \\
\text { católica } \\
\end{array}$ & $\begin{array}{l}\text { Justicia } \\
\text { social }\end{array}$ & Feminista & Feminista \\
\hline $\begin{array}{l}\text { Estructuras } \\
\text { consultivas con el } \\
\text { movimiento de } \\
\text { mujeres }\end{array}$ & $\begin{array}{l}\text { Débil (Consejo } \\
\text { Consultivo con } \\
\text { escaso } \\
\text { funcionamiento) }\end{array}$ & $\begin{array}{l}\begin{array}{l}\text { Fuerte } \\
\text { (múltiples }\end{array} \\
\text { estructuras y } \\
\text { redes) }\end{array}$ & Inexistentes & Débil & \begin{tabular}{|l|}
$\begin{array}{l}\text { Débil } \\
\text { (incipiente }\end{array}$ \\
Consejo \\
Consultivo \\
con ONG \\
en \\
violencia) \\
\end{tabular} & \begin{tabular}{|l} 
Fuerte \\
solamente \\
en violencia \\
(Consejo \\
Consultivo \\
con ONG)
\end{tabular} & $\begin{array}{l}\text { Fuerte } \\
\text { (múltiples } \\
\text { estructuras } \\
\text { y redes) }\end{array}$ \\
\hline CONFIGURACIÓN & Marginal & Insider & Antifeminista & Simbólica & Simbólica & Marginal & Insider \\
\hline
\end{tabular}

Fuente: elaboración propia.

Subsecretaría de la Mujer, 1987-1989. En octubre de 1983, Argentina celebró elecciones libres luego de seis años de dictadura militar, resultando ganador Raúl Alfonsín por la Unión Cívica Radical (UCR), quien gobernó hasta julio de 1989. Este período se caracterizó por los desafíos en torno a la estabilidad institucional y la reparación a las violaciones de los derechos humanos. En este contexto, en marzo de 1987 se creó por decreto presidencial la Subsecretaría de la Mujer en la Secretaría de Desarrollo Humano y Familia del Ministerio de Salud y Acción Social (Decreto 280/87). Considerada el primer organismo nacional especializado en la promoción de los derechos de las mujeres, su jerarquía institucional superó con creces las demandas de las mujeres que pedían sólo una Dirección de la Mujer (Bellucci et al., s. f.). Con el objetivo de "propiciar y favorecer todas las instancias de articulación y coordinación entre el Estado y las mujeres como sujetos sociales y políticos plenos", se trató de "una experiencia corta pero muy intensa"'s.

3 Testimonio de Mónica García Finchaboy. En Atschul (2015: 25). 
Alfonsín designó como autoridad a Zita Montes de Oca, quien tenía un manifiesto compromiso feminista. El equipo de trabajo, aproximadamente 40 personas, se reconoció feminista. Muchas fueron, inicialmente, asesoras ad honorem y posteriormente personal de planta, habiendo trabajado con un enorme "voluntarismo" y recursos escasos (Bellucci et al., s. f.). Esta primera experiencia tuvo una impronta feminista, confrontando la reducción de los temas de "la mujer" a la asistencia social. Se posicionaron las necesidades de las mujeres sin el agregado de "familia", jerarquizando a las mujeres como sujetos de derecho y adoptando la perspectiva de intereses estratégicos de género. Esto se reflejó en la noción de "democratización de las familias como condición básica hacia una igualdad real para las mujeres"4 Se creó el Programa Nacional de Prevención de la Violencia Doméstica, sensibilizando a la policía y a efectores sociales, así los gobiernos provinciales y a ONGs (Ministerio de Relaciones Exteriores y Culto, 1994). Respecto de la articulación federal, con el proyecto de Fortalecimiento de Sectores Sociales (Programa Redes) en cinco provincias, se apuntó a las capacidades institucionales provinciales.

De todas formas, la gestión transversal de las políticas de género se vio entorpecida por el insuficiente reconocimiento institucional de la Subsecretaría. Así las cosas, la Subsecretaría de la Mujer permaneció marginal, al no lograr mayor alcance en la adopción de las políticas de género y porque, además, la estrategia de diálogo definida con las organizaciones de mujeres, mediante el Consejo Consultor, tuvo escaso funcionamiento.

Consejo Nacional de la Mujer, 1991-1994. Por la situación económica y la aguda inflación, Alfonsín adelantó la entrega del mando presidencial a Carlos Saúl Menem en julio de 1989, tras el triunfo del peronismo en las elecciones nacionales. Con dos mandatos de gobierno por su reelección en 1995, se registraron dos momentos marcadamente diferentes con el Consejo Nacional de la Mujer (CNM): el feminismo de Estado primero y, posteriormente, una institucionalidad antifeminista.

El 8 de marzo de 1991 se creó el Consejo Coordinador de Políticas Públicas para la Mujer (Decreto 378/91), dependiente de la Presidencia de la Nación. Su mandato era responder a los compromisos asumidos con la ratificación de la CEDAW que había sido aprobada en 1985 y hacer operativo el Plan de Acción Mundial de Naciones Unidas para el cumplimiento de los objetivos del Decenio Internacional de la Mujer. El Consejo Coordinador

Testimonio de Zita Montes de Oca. En Atschul (2015: 28). 
careció de presupuesto propio y sus funcionarios eran ad honorem. A pesar de las debilidades en los recursos, el Consejo conformó una exitosa Comisión Asesora de partidos políticos ${ }^{5}$ en la que se apoyó la movilización de las mujeres para la aprobación de las cuotas electorales de género en noviembre de 1991 (Ley 24.012).

En 1992, el Consejo Coordinador fue remplazado por el Consejo Nacional de la Mujer (CNM), cuya presidenta tuvo rango de Subsecretaria (Decreto 1426/92). Su mandato era dar cumplimiento a los compromisos de la CEDAW, garantizar la adecuada reglamentación e implementación del cupo femenino, colaborar con las jurisdicciones federales con políticas de género y desarrollar un plan de igualdad de oportunidades. Menem designó a Virginia Franganillo como presidenta. Reconocida feminista con lazos internacionales, ella provenía del peronismo de la Capital Federal y tuvo un papel activo durante la discusión del cupo femenino. Al momento de su designación, integraba la Secretaría de la Función Pública y era jefa de Gabinete del Instituto Nacional de Administración Pública, en cuyo ámbito funcionaba el Programa Estado de la Mujer para la igualdad de género en el empleo público. El CNM tuvo presupuesto propio (Waylen, 2000) y el personal resultó de la redistribución de cargos y concursos, posibilitando el acceso de feministas a cargos ejecutivos (Barrancos, 2007). El CNM contó con un Directorio y el Consejo Federal de la Mujer. Se contó, además, con un Gabinete de Consejeras Presidenciales en políticas de género, creado en 1993 y presidido por el CNM (Decreto 84/93).

El CNM estableció un Plan de Igualdad de Oportunidades para las Mujeres (1993-94) que actuó de paraguas para albergar once programas. Este plan reflejó concepciones relacionales de género, de acciones afirmativas e igualdad de oportunidades, fundamentándose en el feminismo español. El CNM contribuyó con debates centrales respecto de los derechos de las mujeres en las áreas de violencia, acoso sexual y educación. La abogacía para la incorporación de la CEDAW en el plexo de la Constitución de 1994 fue exitosa, y se consagraron previsiones respecto de la igualdad y la no discriminación por género en el capítulo Nuevos Derechos y Garantías.

El CNM impulsó el Programa de Fortalecimiento Institucional de las Áreas Mujer provinciales que permitió la instalación de Consejos Regionales. La articulación con organizaciones de mujeres fue variada y hubo espacios consultivos con la sociedad civil, las mujeres políticas y las sindicalistas. El CNM conformó Comisiones Asesoras, con carácter indicativo y no vinculante, como las Comisiones de Representantes de Mujeres de los Par-

Franganillo en Diario Digital Femenino (2020). 
tidos Políticos, la de Mujeres Empresarias y la de Mujeres Jurídicas - esta última fundamental para la implementación de la Ley de cuotas (Ministerio de Relaciones Exteriores y Culto, 1994). En salud sexual y reproductiva, se conformó un grupo de legisladoras nacionales de todos los partidos (Franganillo, 2019). Se apoyaron organizaciones sociales de mujeres, aprovechando la germinación de organizaciones de base por la situación económica (Barrancos, 2007) y la creciente conformación de ONGs.

Consejo Nacional de la Mujer, 1994-1999. El debate en torno a la reforma constitucional en 1994 cambió el destino del CNM. La propuesta oficialista de incluir una cláusula que prohibiría cualquier forma de aborto (Bellucci, 1997) detonó la renuncia de Franganillo y, con su salida, la de otras feministas (Waylen, 2000). Lopreite (2015) señala este hito como el giro familista del CNM, es decir, la mirada sobre las mujeres de hogares pobres como merecedoras de asistencia social solamente en calidad de madres, desconociendo sus derechos y necesidades, particularmente las de salud sexual y reproductiva. Esther Schiavoni, peronista autoidentificada 'menemista', fue designada como presidenta en 1996. Declarándose defensora de la familia y del "derecho a la vida", con adscripción al centro -derecha político, era una opositora a los derechos sexuales y reproductivos (Waylen, 2000). Se eliminó el Gabinete de Consejeras Presidenciales (Decreto 291/95), por lo que las decisiones quedaron concentradas en Schiavoni.

El CNM se convirtió en una agencia cercana al antifeminismo y aislada del movimiento de mujeres. Además de centrarse exclusivamente en algunos de los intereses prácticos de las mujeres, lo hizo bajo interpretaciones restrictivas en términos de derechos. Esta reversión de los marcos de sentido produjo un distanciamiento del movimiento de mujeres y bloqueó los procesos de incidencia (Weathers, 2004). La cercanía del CNM con el presidente redundó en su deslegitimación, escasa autonomía funcional y alta dependencia presupuestaria para las acciones en violencia. No se avanzó con el Plan de Igualdad de Oportunidades 1995-1999, si bien el CNM monitoreó el cumplimiento de la ley de cupo y desarrolló acciones en violencia (Gobierno de la República Argentina, 1999).

Consejo Nacional de las Mujeres, 2003-2007. Las elecciones presidenciales de 1999 ungieron a Fernando De La Rúa como presidente de la Alianza para la Producción, el Trabajo y la Educación, pero la crisis económica y las medidas de ajuste culminaron con su renuncia en diciembre de 
2001. Luego de sucesivos presidentes interinos, en 2002, Eduardo Duhalde, del Partido Justicialista, fue designado en dicha función para convocar a elecciones nacionales.

En 2003, el peronista Néstor Kirchner resultó electo como presidente por el Frente para la Victoria, inaugurando el período conocido como kirchnerismo hasta 2015, ya que fue seguido por dos presidencias consecutivas de su esposa Cristina Fernández (2007-2011 y 2011-2015). Este gobierno posicionó al Estado como garante de derechos políticos, económicos y sociales, privilegiando políticas redistributivas. En el interregno desde la asunción de Duhalde como presidente interino y el ascenso de Néstor Kirchner al Poder Ejecutivo en 2003, la jerarquía del CNM ya se había diluido bajo el Consejo Coordinador de Políticas Sociales (Decreto 357/02).

Néstor Kirchner mantuvo el CNM en el Consejo Coordinador de Políticas Sociales. En 2004 asumió María Lucila "Pimpi” Colombo como presidenta. Peronista católica y contraria a la despenalización del aborto, era fundadora y activista de Sindicato de Amas de Casa. Colombo priorizó la asistencia social a las familias en condiciones de pobreza sin incorporar los derechos sexuales y reproductivos (Carbajal, 2008).

Según Lopreite (2015), Colombo continuó el enfoque familista que desestimó las jerarquías y desigualdades de género que se reproducen en los hogares. Las medidas buscaron rescatar los "saberes tradicionales" de las mujeres (Bedford, 2009). En la implementación de programas territoriales, Colombo convocó a organizaciones comunitarias, sin experiencia en género, al punto que Bedford (2009) menciona un proceso de "recatolización" de la provisión de servicios sociales. La comunicación con los movimientos de mujeres y las feministas en particular fue acotada, introduciendo una situación de distanciamiento institucional.

Consejo Nacional de las Mujeres, 2008-2015. Con Cristina Fernández, entre 2008 y 2011, Lidia Mondello ejerció como presidenta. Era una activista católica de base, pero más abierta que su predecesora en materia de educación sexual, y derechos sexuales y reproductivos. Abogó por la adecuada implementación del aborto legal, confrontando públicamente al Poder Judicial por impedir que una adolescente violada interrumpa su embarazo. Durante su gestión, Mondello activó el Directorio del CNM y éste, en 2010, pasó a denominarse Consejo Nacional de las Mujeres en plural (Decreto 326/10).

Entre 2011 y 2015, Mariana Grass ejerció la presidencia del CNM, bajo un nuevo marco normativo dado por la Ley 26.485 de "Protección integral 
para prevenir, sancionar y erradicar la violencia contra las mujeres en los ámbitos en que desarrollan sus relaciones interpersonales" aprobada por el Congreso en 2009 y reglamentada en 2010 (Decreto 1011/10). Esta ley posicionó al CNM como el organismo rector en materia de políticas de violencia de género, bajo un enfoque de derechos humanos siguiendo la Convención de Belém Do Pará. En cumplimiento con la normativa mencionada, el CNM puso en marcha el Plan Nacional de Acción 2014-2016 para la prevención, asistencia y erradicación de la violencia contra las mujeres y se conformó la Red Nacional de Violencia de Género. Asimismo, se instaló el Observatorio de Violencia de Género y la línea telefónica para la atención en violencia (línea 144).

Grass carecía de formación feminista y de vínculos con el movimiento. El bajo presupuesto del CNM no le parecía problemático porque las "políticas" (y no los recursos) deberían ser objeto de controversia (Tabbush et al., 2019). Grass priorizó las "injusticias sociales" sin necesariamente considerar cómo estas se reproducen diferencialmente para mujeres y hombres.

Grass incorporó algunas feministas al CNM en la gestión, precisamente en asuntos de violencia y en las capacitaciones para acceder a pequeños subsidios (Programa Ellas Hacen) (Rodríguez Gustá, 2018). Pero su gestión no privilegió el funcionamiento de estructuras consultivas con los movimientos de mujeres y feministas que, en esos años, estaban logrando escala. El Plan Nacional de Violencia, reclamado por el movimiento de mujeres, debía anunciarse y ponerse en marcha en 2011. Recién se lo hizo público en 2015, después de la multitudinaria marcha "Ni Una Menos" en contra de la violencia hacia las mujeres y el femicidio, evento que mostró la insuficiencia del nexo entre el CNM y los movimientos sociales.

En suma, el CNM comenzó a abordar intereses estratégicos de género, aunque limitados a violencia. Se mantuvo como simbólico para la acción feminista, al punto que reconocidas referentes feministas se vincularon con otros segmentos del Estado e incluso con el Congreso para avanzar con legislación, programas y servicios en educación sexual, igualdad de oportunidades en el trabajo, identidad de género y matrimonio igualitario (Rodríguez Gustá et al., 2017).

Instituto Nacional de las Mujeres, 2015-2019. En 2015 se produjo un significativo cambio de gobierno con la elección presidencial de Mauricio Macri por Propuesta Republicana (PRO), un partido de centro derecha que ganó las elecciones presidenciales bajo la Alianza Cambiemos con la UCR. Esto trajo una jerarquización del CNM en 2017, con la creación del Institu- 
to Nacional de las Mujeres (INAM), manteniendo el perfil especializado en violencia (Decreto 698/17). El INAM, como ente descentralizado en la jurisdicción del Ministerio de Desarrollo Social de la Nación, ganó autonomía en la toma de decisiones y ejecución de su presupuesto. A fines de 2019, el INAM contaba con 260 personas, pero sufrió restricciones presupuestarias por la inflación y los ajustes fiscales del gobierno.

Fabiana Túñez, feminista con destacada trayectoria en violencia, fundadora y presidenta de la ONG Casa del Encuentro (pionera en relevar el número de femicidios según la prensa en ausencia de un registro oficial unificado), fue designada presidenta. Favorable al aborto, Túñez utilizó el pañuelo verde de la Campaña por el Aborto Legal, Seguro y Gratuito durante la discusión del proyecto de ley en el Congreso en 2018.

El INAM elaboró el Plan de Igualdad de Oportunidades y Derechos 2018-2020, pero con escasa incidencia del movimiento de mujeres. Con una perspectiva relacional de género, este plan se enmarcó en el enfoque de derechos, en la interseccionalidad de las desigualdades de género y en las políticas de transversalización (INAM, 2018), para avanzar, más plenamente, con los intereses estratégicos de género. Además, se puso en marcha el Plan Nacional para la Prevención, Atención y Erradicación de la Violencia contra la Mujer y se ampliaron los servicios de atención (Lopreite y Orsino, 2017). La sanción de la Ley Micaela 27.499 (2019) de capacitación obligatoria en género y violencia contra las mujeres en el Estado, con el INAM como órgano rector, fortaleció su gravitación.

Este Instituto regularizó el funcionamiento del Consejo Federal de Mujeres que permitió consolidar el nexo con las provincias y los municipios. Mantuvo vínculos con organizaciones sociales en el Consejo Consultivo ad honorem en violencia, garantizando al menos dos organizaciones por provincia. Algunas ONG feministas valoraron la jerarquización del INAM como respuesta a sus demandas y monitorearon su presupuesto, en ocasiones denunciando incumplimientos ante el Congreso (Página 12, 2017, p. 12). Sin embargo, los nexos fueron a todas luces insuficientes para un movimiento feminista expandido y múltiple, manteniendo el distanciamiento institucional que también caracterizó al CNM en la gestión anterior.

Ministerio de las Mujeres, Género y Diversidad, 2019-2021. En un contexto de inestabilidad financiera e inflación, en 2019 resultó triunfante el peronista Alberto Fernández, por el Frente de Todos. Fernández creó el Ministerio de las Mujeres, Género y Diversidades (MMGyD) cuya normativa estableció los derechos de las mujeres y las diversidades como "objetivos 
prioritarios" del Estado (Decretos 7/2019 y 50/2019). El MMGyD cristaliza un feminismo de Estado que adopta la agenda transfeminista de la época, con demandas propiamente interseccionales cuya naturaleza es incorporar las necesidades de género, con identidades y preferencias sexo-genéricas, según clase, raza, generación y condiciones territoriales. Elisabeth Gómez Alcorta, abogada especializada en derechos humanos y pueblos originarios y activista social, fue designada ministra. Autodefinida como "una militante que trabaja de ministra" (Carbajal, 2019), privilegió transversalizar y federalizar las políticas de género en la gestión gubernamental.

Con la jerarquización institucional, fue posible aumentar la dotación de personal, contabilizando 700 personas a mayo de 2021 (Carbajal, 2019). Se instituyó el Gabinete Nacional para la Transversalización de las Políticas de Género (GNTPG), presidido por el Jefe de Gabinete de Ministros e integrado por las autoridades de los ministerios y las restantes entidades públicas federales (Decreto 682/2020). Por la relevancia y especificidad de la violencia de género, el MMGyD conformó el Consejo Federal para la Prevención y el abordaje de femicidios, travesticidios y transfemicidios (Decreto 123/2021). Para la coordinación con los gobiernos subnacionales, el Consejo Federal del Ministerio de las Mujeres, Géneros y Diversidad de la Nación, de carácter ad honorem, con las áreas provinciales con competencia en género, igualdad y diversidad.

El MMGyD cuenta con dos planes rectores con lenguaje propio de intereses estratégicos de género. El más emblemático es el Plan Nacional de Acción contra las Violencias por Motivos de Género 2020-2022 el cual, debido a la pandemia del COVID-19 y las medidas de restricción, ganó relevancia en la prevención y la ampliación de los centros integrales y territoriales de atención, incluyendo apoyo económico. Por su parte, el Plan Nacional de Igualdad en la Diversidad (2021-2023) que se presentó como "interseccional, federal y participativo" propone crear condiciones de igualdad sustantiva para que las mujeres y LGBTI+ puedan acceder a sus derechos y ejercerlos de manera libre, justa e igualitaria.

Respecto de su vinculación con organizaciones sociales, el MMGyD jerarquizó los procesos consultivos y las redes territoriales. Esto se reflejó en un Consejo Asesor Ad Honorem con activistas provenientes de ámbitos académicos, sindicales, de los movimientos sociales, organizaciones sociales, asociaciones y colegios de profesionales, con recomendaciones no vinculantes (Resolución 24/2020). También desarrolló redes con otras mujeres de ámbitos institucionalizados, lo cual fue fundamental en el proceso de elaboración y aprobación de la despenalización del aborto, en diciembre de 2020 por el Congreso (Ley 27.610). 


\section{Demandas feministas, estrategias gubernamentales y configuraciones institucionales}

Recapitulando, la Subsecretaría de la Mujer fue una agencia marginal, pero seguidamente, el CNM se transformó en Insider con un fuerte nexo con el movimiento de mujeres. Sin embargo, esto fue de corta duración, ya que el CNM rápidamente se volvió una agencia antifeminista y antagónica al movimiento de mujeres. Durante el kirchnerismo el CNM fue una agencia simbólica, por lo que no recuperó su otrora impronta de feminismo de Estado. El INAM, a pesar de la jerarquización institucional, fue marginal. Fue con el MMGyD que se recuperó el estatus de agencia Insider para el movimiento de mujeres y los colectivos LGBTI+.

Ahora bien, ¿qué factores explican estas variaciones en las expresiones institucionales de la agencia para las mujeres? Por ejemplo, ¿qué razones dan cuenta del pasaje rápido de Insider a antifeminista bajo un mismo presidente? ¿Por qué razones el CNM se mantuvo simbólico mientras se avanzaba en legislaciones innovadoras en derechos de mujeres, como lo ejemplifica el kirchnerismo? ¿Por qué la agencia fue marginal tanto en el gobierno de la UCR como de Cambiemos, a pesar de los disímiles entornos sociopolíticos? ¿Qué factores llevaron a la creación de un ministerio como agencia Insider? Para armar el rompecabezas, proponemos examinar la estructura y dinámica del movimiento de mujeres y las estrategias de negociación del Poder Ejecutivo.

La creación de la Subsecretaría resultó de la puesta en agenda por parte del movimiento de mujeres de la necesidad de un organismo rector en materia de derechos de mujeres. La alianza de mujeres políticas, de diferentes extracciones partidarias y sindicales denominada Multisectorial de Mujeres, hizo pública su plataforma el 8 de marzo de 1984. Entre otros puntos, instaron a la creación de una Secretaría de Estado de la Mujer (Tarducci, 2012). Asimismo, el entorno de ideas favorable con la nueva legislación civil otorgando a las mujeres derechos sobre sus hijos (Ley 23.265, Patria Potestad Compartida en 1985) y el Divorcio Vincular (Ley 23.515, 1987), propició un marco de sentidos afín al desarrollo de institucionalidad estatal.

Asimismo, el naciente régimen global de género ofreció oportunidades para la organización y el armado de redes de mujeres, a la luz del Decenio de la Mujer de las Naciones Unidas y la ratificación de la CEDAW por parte del Congreso nacional (Ley 23.179) en 1985. Las reuniones preparatorias y la Conferencia de Nairobi permitieron el desarrollo de redes que llevaron al primer Encuentro Nacional de las Mujeres en 1986 que se constituiría como semillero del movimiento en el país (Alma y Lorenzo, 2013) . 
Por cierto, existía un compromiso del presidente Alfonsín quien prometió en el primer Día Internacional de la Mujer una Dirección de la Mujer (Bellucci et al., s. f.). La UCR era un partido de centro influido por la social democracia de la época. A la luz la democratización, el Estado debía abrirse a diferentes actores y abordar las demandas sociales y la Subsecretaría fue un reflejo de este proceso. Por ende, la plataforma programática del Poder Ejecutivo fue un factor habilitante para el desarrollo de una agencia de políticas de mujeres.

La Subsecretaría, sin embargo, no logró desarrollar el nexo de interlocución con el movimiento de mujeres y la presencia de feministas resultó insuficiente. El Estado todavía tenía el manto de sospecha de aparato represivo y coercitivo (Bellucci et al., s. f.). La composición excesivamente numerosa del Consejo Consultivo y la falta de claridad en los roles interfirió con su buen funcionamiento (Atschul, 2015). Las peronistas pronto giraron su acción hacia el Consejo de las Mujeres de la Provincia de Buenos Aires, ya que, en un contexto de debilitamiento del gobierno nacional por la alta inflación, la Subsecretaría perdió atractivo para la acción política.

El CNM como agencia Insider es fruto del movimiento de mujeres políticas y, especialmente, de las peronistas de la Capital Federal, cuyas ideas programáticas tomaban elementos del feminismo español y brasileño (Franganillo, 2019). El CNM se conformó con destacadas feministas de diferentes partidos políticos. La "condición" fue su identidad feminista, con formación académica y experiencia de gestión pública o en el ámbito social. Algunas de ellas provenían del Consejo Provincial de la Mujer, por lo que aportaron sus propias redes territoriales. Esta cualidad no partidaria pero feminista "fue una de las tantas fortalezas que tuvo el Consejo" 6 para vincularse con el movimiento de mujeres más amplio.

Asimismo, la movilización de mujeres en torno al debate y la aprobación del cupo electoral femenino (ley 24.012 en 1991) generó alianzas políticas multipartidarias y esto facilitó el respaldo de organizaciones sociales de mujeres. En 1990, las participantes del Encuentro Nacional de Mujeres avalaron el cupo femenino y nació la Red de Feministas Políticas, integrada por mujeres de más de quince partidos, comprometidas con la transformación de la representación de las mujeres en el Estado (Caminotti, 2016).

La articulación con las mujeres políticas fue una característica del CNM que facilitó el nexo con el movimiento social. El CNM promovió una red con legisladoras de diferentes partidos para conformar un grupo de trabajo en salud sexual y reproductiva (Franganillo, 2019). Al momento de la Con-

6 Entrevista con Virgina Franganillo. Buenos Aires, 2018. 
vención Constituyente, esta red posibilitó sinergias con más de cien organizaciones en MADEL (Mujeres Autoconvocadas por el Derecho a Decidir el Libertad (Sutton y Borland, 2013), al calor del V Encuentro Feminista Latinoamericano y del Caribe realizado en Argentina (1990) ${ }^{7}$.

El CNM abrió espacios consultivos nacionales y regionales con la sociedad civil. El Consejo Federal de la Mujer buscó emular el Consejo Nacional de los Derechos de la Mujer de Brasil que incluía a las organizaciones sociales en los procesos decisorios ${ }^{8}$. Pero, además, las intervenciones fomentaron la organización, agregación y movilización de intereses de las mujeres, particularmente en los ámbitos provinciales. El nexo con el movimiento de mujeres contó, además, con una red de femócratas, en el entorno de 3.000 funcionarias sensibilizadas en género del Programa Estado y Mujer 9 .

Para Menem, quien accedió al Poder Ejecutivo en medio de turbulencias sociales y económicas y con una propuesta privatizadora, el apoyo a la movilización de las mujeres por la participación política fue una forma de ganar adhesiones sin afectar temáticas sensibles. Esta misma instrumentalidad puso al CNM en la regulación de tensiones con sus aliados conservadores, grupos islámicos y el catolicismo. La conversión del CNM a una agencia antifeminista obedeció a la coyuntura política de toma de posición en derechos sexuales y reproductivos, a la luz de la Conferencia Internacional de Población y Desarrollo (CIPD) (1994) y la Cuarta Conferencia Mundial de la Mujer en Beijing (1995). La reacción conservada se vio reflejada, además del cambio en el CNM, en las reservas al Plan de Acción de El Cairo y en el alineamiento con el Vaticano en la Conferencia de Beijing. El movimiento de mujeres no logró revertir el retroceso del CNM, pero comenzaron a consolidarse ONGs especializadas en derechos de mujeres y, en el auge del régimen global de género, se propiciaron redes e intercambios supranacionales (Lopreite, 2010a).

Bajo Néstor Kirchner, el CNM en tanto agencia simbólica resultó de una estrategia de regulación política en momentos en que urgía asegurar la gobernabilidad por la aguda crisis económica y social. Kirchner asumió en 2003 con un $22 \%$ del electorado y necesitaba construir una coalición en la que los movimientos de trabajadores desempleados (piquetereos) fueron aliados (Etchemendy y Garay, 2011). Los piqueteros priorizaban el desempleo y las condiciones básicas de vida, por lo que las disputas de género eran una distracción sobre los temas distributivos (Cross y Freytes Frey, 2007). El CNM

\footnotetext{
7 En este Encuentro se había declaró el 28 de setiembre como el Día por la Despenalización del Aborto en la región.

8 Franganillo en Diario Digital Femenino (2020).

9 Entrevista con Virgina Franganillo. Buenos Aires, 2018.
} 
fue, además, un espacio negociado para Duhalde, quien colaboró con el posicionamiento de la candidatura presidencial de Kirchner en el peronismo. Desde su gestión como gobernador de la Provincia de Buenos Aires, Duhalde promovió un enfoque familista de la política social con contenidos cristianos, donde las mujeres de bajos ingresos eran "sostén" familiar (Zaremberg, 2004). Finalmente, el carácter simbólico del CNM también reguló la incómoda relación entre los Kirchner y la Iglesia (Tabbush et al., 2019), cuyo protagonismo se había expandido con el Diálogo Político armado por Duhalde durante la crisis del 2001 que Kirchner buscó acotar.

Los nexos entre el Estado y los movimientos de mujeres y las feministas no se desarrollaron con el CNM, sino con legisladoras feministas del Congreso y con femócratas al frente de otros organismos del Estado, en las áreas de trabajo, salud y educación, por ejemplo (Rodríguez Gustá et al., 2017). Estos canales de influencia por fuera del CNM amortiguaron conflictos con actores clave para el gobierno porque los desarrollos, se orientaron a temáticas especializadas y sectoriales. Por cierto, estas redes produjeron nuevas normativas como la de violencia de género la que posibilitó, de forma indirecta, un cambio en el propio $\mathrm{CNM}$, facilitando la adopción de intereses estratégicos de las mujeres en las intervenciones de violencia.

En 2017, cuando nace el INAM, el movimiento de mujeres estaba masificado con renovación de clase y generacional y más propiamente "feminista" en sus demandas (Beckwith, 2007). Las mujeres participantes de los Encuentros Nacionales de Mujeres fueron aumentado en número y en algunos de ellos se sentaron las bases para el surgimiento de la Campaña Nacional por el Derecho al Aborto Legal Seguro y Gratuito, como en Rosario (2003) y Mendoza (2004) (Sutton y Borland, 2013). Las mujeres de sectores populares habían avanzado con demandas feministas e incluso influido en los movimientos de trabajadores desocupados (Di Marco, 2011). Asimismo, la creciente sensibilidad social respecto de los feminicidios, junto con el expresivo crecimiento de la Campaña por el aborto legal, especialmente entre las jóvenes, llevó a la masiva marcha "Ni Una Menos" en junio de 2015. Este evento marcó un punto de inflexión en la incidencia, buscada o no intencional, sobre la agencia de las mujeres. Con los temas de igualdad de género en la agenda pública y un actor social con una presencia callejera sin paralelo, se produjo una fuerte presión sobre la institucionalidad pública para dar mayores respuestas.

Macri ganó en segunda vuelta y careció de mayoría legislativa. La creación del INAM recogió una demanda de erradicar la violencia contra las mujeres, instalada masivamente desde 2015. Sin embargo, las erogaciones presupuestarias fueron insuficientes y los nexos con los movimientos de 
mujeres quedaron confinados a esta temática, quedándose sin interlocutoras en economía y derechos sexuales y reproductivos, asuntos salientes del movimiento social de mujeres. Asimismo, la parte del movimiento ligado al peronismo y a los partidos de izquierda manifestó su oposición a las medidas económicas del gobierno de Cambiemos, distanciándose del INAM por considerarlo ubicado en una posición contradictoria sin posible resolución y, por lo tanto, no representativo de los intereses estratégicos de las mujeres.

El MMGyD en tanto agencia Insider responde al avance del feminismo en tanto actor político que logró teñir la contienda electoral presidencial de 2019. A raíz del debate legislativo del aborto en 2018, las plataformas partidarias debieron abordar este tema que ganó predominancia en la agenda de género. El candidato Alberto Fernández se comprometió durante la campaña electoral a impulsar la legalización del aborto y la creación de un ministerio. El nuevo Poder Ejecutivo fue fundamental en esta construcción del feminismo de Estado.

El MMGyD resultó de la intensa movilización en torno a la despenalización del aborto. Cuando los actores políticos avanzaron con la propuesta de creación de un ministerio, el movimiento feminista ya tenía una escala masiva sin precedentes, con un feminismo joven y popular centrado en aborto y en demandas queer, claramente identificado con el kirchnerismo como expresión política (Sutton, 2020). A esto se sumó una expresiva presencia de femócratas en ámbitos institucionales (el propio Estado, universidades, partidos políticos y sindicatos). El recorrido del movimiento de mujeres y feministas, incluyendo su ensanchamiento y amplitud debido a la incorporación de sectores con más amplias y renovadas perspectivas, como las identidades de género y diversidad sexual, conforman un actor social con demandas muy fuertes de soslayar sentando las bases de una agencia Insider.

\section{Conclusiones}

La robustez institucional de las agencias para las mujeres es relevante en tanto es una manifestación de la capacidad estatal para abordar las problemáticas de la desigualdad de género. Este trabajo mostró la construcción de la capacidad estatal en función del modelo aspiracional del Feminismo de Estado. La institucionalidad de género se instala y desinstala con facilidad y por lo tanto está menos consolidada que lo que podría esperarse de los estudios de género e institucionalismo, cuyo énfasis ha sido mostrar la cristalización de la agenda de mujeres (Guzmán, 2019). Esto no significa negar 
la existencia de experiencias de feminismo de Estado en América Latina, sino que, como sugiere el caso argentino, se trataría de un proceso dinámico sujeto a cambios incluyendo avances y retrocesos, con configuraciones oscilantes entre agencias marginales o incluso simbólicas, y otras Insiders. Estas distintas configuraciones, a su vez, son resultado de varios factores. En esta investigación privilegiamos la movilización feminista alrededor de los temas de género y también las decisiones estratégicas del Poder Ejecutivo al intentar responder, ignorar o incluso bloquear las demandas feministas.

En Argentina, el análisis diacrónico mostró la prevalencia de las agencias marginales y simbólicas, con que un breve intervalo claramente antifeminista. Las dos coyunturas con agencias Insiders ocurrieron en momentos históricos inmediatamente posteriores a picos de movilización feminista (participación política y aborto respectivamente). El hecho de que existió un precedente de agencia Insider, pero que rápidamente cambió, sugiere que la consolidación de una institucionalidad de feminismo de Estado puede truncarse si los factores involucrados en su desarrollo sufren modificaciones evidentes. En este sentido, las decisiones estratégicas de los gobernantes frente a coyunturas políticas y económicas donde inciden grupos conservadores resultan determinantes a la hora de los cambios en la institucionalidad.

Estos hallazgos muestran, por un lado, la relevancia del movimiento de mujeres y la construcción de demandas feministas ya que se trata del actor que coloca en la agenda pública la necesidad de modificar las estructuras de autoridad del Estado y, en particular, de crear y consolidar agencias para las mujeres. Por otro lado, se reafirma la ausencia de una asociación directa entre ideología gubernamental y consolidación de estas agencias. Como se examinó, las diversas coyunturas críticas, la necesidad de los líderes de expandir sus bases de apoyo y de incorporar demandas sectoriales de alta intensidad y relevancia electoral abonan al uso instrumental que el Poder Ejecutivo pueda hacer de los asuntos de género. Lo que sí resulta clave es la intersección entre un movimiento feminista robusto y con nexos institucionalizados con el partido gobernante para verificar la aparición de agencias Insider en coyunturas de menor saliencia de actores conservadores.

Para finalizar, este artículo contribuyó con el análisis de la configuración de una agencia de alcance nacional en un sistema federal como el de Argentina. Es importante resaltar que, en la medida en que las investigaciones sobre feminismo de Estado se concentran en el nivel de los Estados nacionales, para el caso argentino como el de otras federaciones, una dimensión relevante del poder de estas agencias estará dada por su capacidad de influir en las políticas públicas de género más allá del nivel nacional. 


\section{Referencias bibliográficas}

Alma, A. y Lorenzo, P. (2013). Mujeres que se encuentran. Una recuperación histórica de los Encuentros Nacionales de Mujeres en Argentina (1986-2005). Yegua Ediciones.

Alvarez, S. E. (2009). Beyond NGO ization?: Reflections from Latin America. Development, 52(2), 175-184.

Astelarra, J. (2004). Políticas de género en la Unión Europea y algunos apuntes sobre América Latina. CEPAL.

Atschul, M. (2015). "Zita Montes de Oca: La funcionaria y la feminista”. En S. Pérez Gallart \& A. M. Ubeira (Eds.), Las mujeres conquistando derechos en los 30 años de democracia (pp. 23-36). APDH. Asamblea Permanente por los Derechos Humanos e INADI.

Barrancos, D. (2007). Mujeres en la sociedad argentina. Una historia de cinco siglos. Buenos Aires: Sudamericana.

Beckwith, K. (2007). Mapping Strategic Engagements: WOMEN'S MOVEMENTS AND THE STATE. International Feminist Journal of Politics, 9(3), 312-338.

Bedford, K. (2009). Developing partnerships: Gender, sexuality, and the reformed World Bank. Minnesota: University of Minnesota Press.

Bellucci, M. (1997). Women's struggle to decide about their own bodies: Abortion and sexual rights in Argentina. Reproductive Health Matters, 5(10), 99-106.

Bellucci, M., Luvecce, C., Mariani, S., \& Rofman, A. (s. f.). A manera de balance. La Subsecretaría de la Mujer de Argentina (1987-1990). Revista Doxa, 38-45.

Bohn, S. R. (2009). Bridging State and Civil Society? The Amphibious Nature of "State. Feminism" in Brazil. American Political Science Association Meeting, Toronto, Canadá.

Bohn, S. R. (2010). Feminismo estatal sob a presidência Lula: O caso da Secretaria de Políticas para as Mulheres. Revista Debates, 4(2), 81-106.

Çăgatay, N., Grown, C., y Santiago, A. (1986). The Nairobi Women's Conference: Toward a Global Feminism? Feminist Studies, 12(2), 401-412. J

Caminotti, M. (2016). "Cuotas de género y paridad en la legislación electoral de América Latina: Mujeres, partidos políticos y Estado”. En F. Freidenberg y B. Muñoz-Pogossian (Eds.), Reformas a las organizaciones de partidos en América Latina (1978-2015). (pp. 183-203). PUCP, OEA, IIJ-UNAM y SAAP.

Carbajal, M. (2008, junio 6). ¿Dónde está el Consejo Nacional de la Mujer? [Prensa digital]. Página 12. Disponible en https://www.pagina12.com.ar/diario/sociedad/ 3-105523-2008-06-06.html

Carbajal, M. (2019, diciembre 14). "Este Ministerio es una conquista del movimiento de mujeres y diversidad y lo voy a construir con todos los sectores" Las pautas que guiarán la gestión de Elizabeth Gómez Alcorta. Página 12. Disponible en https://www.pagina12.com.ar/236597-las-pautas-que-guiaran-la-gestion-deelizabeth-gomez-alcorta 
Cross, C., y Freytes Frey, A. C. (2007). Movimientos piqueteros: Tensiones de género en la definición del liderazgo. Argumentos (MÃ|copyrightxico, D.F), 20(55), 77-94.

Di Marco, G. (2011). El pueblo feminista. Movimientos sociales y lucha de las mujeres en torno a la Ciudadanía. Buenos Aires: Biblos.

Diario Digital Femenino. (2020, agosto 7). A 28 años del Decreto de creación del Consejo Nacional de la Mujer. Diario Digital Femenino. Una cuestión de género. Disponible en: https://diariofemenino.com.ar/df/a-28-anos-del-decreto-decreacion-del-consejo-nacional-de-la-mujer/

Díaz García, V. (2016). Los mecanismos nacionales y regionales de las mujeres en América Latina y el Caribe Hispano. ONU Mujeres.

Eisenstein, H. (1996). Inside Agitators: Australian Femocrats and the State. Temple University Press.

Etchemendy, S., y Garay, C. (2011). “Argentina’s Left Populism in Comparative Perspective". En S. Levitsky y K. M. Roberts, The Resurgence of the Latin American Left (pp. 283-305). Baltimore: Johns Hopkins University Press.

Fernós, M. D. (2010). National mechanism for gender equality and empowerment of women in Latin America and the Caribbean region (Vol. 102). CEPAL.

Franceschet, S. (2003). "State feminism" and women's movements: The impact of Chile's Servicio Nacional de la Mujer on women's activism. Latin American Research Review, 38(1), 9-40.

Franganillo, V. (2019). “La creación del Consejo Nacional de la Mujer”. En E. Díaz (Ed.), Feminismos y personismo. Reflexiones históricas y actuales de una articulación negada (pp. 153-165). La Plata: EDULP / Editorial de la Universidad Nacional de La Plata.

Franganillo, V. (2020). "A 28 años de la creación del Consejo Nacional de la Mujer". Diario Digital Femenino. Disponible en https://diariofemenino.com.ar/df/a-28anos-del-decreto-de-creacion-del-consejo-nacional-de-la-mujer/

Friedman, E. J., y Tabbush, C. (2019). "Introduction. Contesting the Pink Tide". En E. J. Friedman (Ed.), Seeking Rights from the Left. Gender, Sexuality, and the Latin American Pink Tide (pp. 1-47). Durham: Duke University Press.

Gobierno de la República Argentina. (1999). Infrome de la República Argentina. Cuarto Informe Periódico (septiembre de 1996-octubre de 1999). [CEDAW].

Guzmán, V. (2001). La institucionalidad de género en el Estado: Nuevas perspectivas de análisis. CEPAL.

Guzmán, V. (2019). “Prefacio”. En A. L. Rodríguez Gustá (Ed.), Marchas y contramarchas en las políticas locales de género. Dinámicas territoriales y ciudadanía de las mujeres en América Latina (pp. 9-13). CLACSO / Consejo Latinoamericano de Ciencias Sociales.

Hernes, H. (1987). Welfare State and Woman Power. Essays in State Feminism. Norwegian University Press. 
INAM. (2018). Plan de Igualdad de Oportunidades y Derechos (PIOD) 2018-2020. INAM. Kantola, J. y Outshroom, J. (2007). “Changing State Feminism”. En Changing State Feminism (pp. 1-20). Londres: Palgrave Macmillan.

Levy, C. y Bohn, S. (Eds.). (2021). Twenty-First-Century Feminismos Women's Movements in Latin America and the Caribbean. McGill-Queen's University Press.

Lopreite, D. (2010a). “Transnational Activism and the Argentinean Women's Movement: Challenging the Gender Regime?” En D. Caouette, D. Masson y P. Dufour (Eds.), Transnationalizing Women's Movements: Solidarities Without Borders (pp. 127-148). University of British Columbia Press.

Lopreite, D. (2010b). Gender and Welfare Regime Change in Argentina: International Conventions and Feminists Networks in a New Social Agenda. Disponible en http:// citeseerx.ist.psu.edu/viewdoc/download?doi=10.1.1.604.5732\&rep=rep1\&.type $=$ pdf Lopreite, D. (2015). Gender policies in Argentina after neoliberalism: Opportunities and obstacles for women's rights. Latin American Perspectives, 42(1), 64-73.

Lopreite, D. y Orsino, S. (2017). La historia del Instituto Nacional de la Mujer (INAM). Antecedentes, análisis y balance (1983-2017) (p. 26). Instituto Nacional de las Mujeres. Manzano, V. (2019). "Feminismo y juventud en la Argentina del siglo XX". En M. Larrondo \& C. Ponce Lara (Eds.), Activismos Feministas Jóvenes: Emergencias, Actrices y Luchas en América Latina (pp. 41-58). CLACSO.

Mazur, A. G. (2012). "A feminist empirical and integrative approach in political science: Breaking down the glass wall?" En H. Kincaid, The Oxford Handbook of Philosophy of Social Science. Oxford University Press.

McBride, D. E. y Mazur, A. (1995). Comparative state feminism. Sage Publications.

McBride, D. E. y Mazur, A. G. (2010). The Politics of State Feminism: Innovation in Comparative Research. Temple University Press.

Ministerio de Relaciones Exteriores y Culto. (1994). Informe nacional. Situación de la mujer en la República Argentina. Decreto 1370/94.

Miranda, L. y Roque López, B. (2019). "El Mayo Estudiantil Feminista de 2018 en la Pontificia Universidad Católica de Chile "La Revolución es Feminista". En M. Larrondo y C. Ponce Lara (Eds.), Activismos feministas jóvenes: Emergencias, actrices y luchas en América Latina (1a., pp. 59-78). CLACSO. Disponible en http:// biblioteca.clacso.edu.ar/clacso/gt/20191202034521/Activismos-Feministas-Jovenes.pdf

Molyneux, M. (1985). Mobilization without Emancipation? Women's interests, the State, and Revolution in Nicaragua. Feminist Studies, 227-254.

Molyneux, M., y Razavi, S. (2005). Beijing plus ten: An ambivalent record on gender justice. Development and change, 36(6), 983-1010.

Página 12. (2017, septiembre 7). Apoyos y críticas del feminismo. El CNM es Instituto. Página 12. Disponible en https://www.pagina12.com.ar/61351-el-cnm-es-instituto. Richards, P. (2004). Pobladoras, Indigenas and the State: Difference, Equality and Women's Rights in Chile. New Jersey: Editorial Rutgers University. 
Rodríguez Gustá, A. L. (2018). "El Consejo Nacional de la Mujer en la época progresista: Cuando se está lejos del feminismo de Estado". En M. M. Ollier (Ed.), La centenaria apuesta de la Argentina democrática (pp. 245-254). Buenos Aires: Prometeo Libros.

Rodríguez Gustá, A. L. (2021). Women's policy agencies and government ideology: The divergent trajectories of Argentina and Brazil, 2003-2019. International Feminist Journal of Politics, 23(4), 625-647.

Rodríguez Gustá, A. L., Madera, N. y Caminotti, M. (2017). Governance Models of Gender Policy Machineries under Left and Right Governments in Latin America. Social Politics: International Studies in Gender, State E Society, 24(4), 452-480.

Sutton, B. (2020). Intergenerational encounters in the struggle for abortion rights in Argentina. Women's Studies International Forum, 82, 102392.

Sutton, B. y Borland, E. (2013). Framing Abortion Rights in Argentina's Encuentros Nacionales de Mujeres. Feminist Studies, 39(1), 194-234.

Tabbush, C., Díaz, M. C., Trebisacce, C. y Keller, V. (2019). "LGBT Rights Yes, Abortion No: Explaining Uneven Trajectories in Argentina under Kirchnerism (200315)". En E. J. Friedman (Ed.), Seeking Rights from the Left: Gender, Sexuality, and the Latin American Pink Tide (pp. 82-114). Durham: Duke University Press.

Tarducci, M. (2012). La lucha de las mujeres por el derecho al aborto en Argentina. Primera Jornada Argentina y Latinoamericana de la Asociación de Medicina Psicosocial Argentina: "Lo visible y lo invisible del aborto", Ciudad Autónoma de Buenos Aires.

Waylen, G. (2000). Gender and Democratic Politics: A Comparative Analysis of Consolidation in Argentina and Chile. Journal of Latin American Studies, 32, 765-793.

Weathers, G. J. (2004). Argentina and Chile: Politics and Fronteras in Geographies of Gender and Nation [Tesis doctoral]. University of Maryland.

Weldon, S. L. (2002). Beyond Bodies: Institutional Sources of Representation for Women in Democratic Policymaking. The Journal of Politics, 64(4), 1153-1174.

Zaremberg, G. (2004). Alpargatas y libros: Estilos de gestión, género y política social en Argentina y Chile (Vol. 90). United Nations Publications.

Zaremberg, G., y Almeida, D. R. de. (2021). Blocking anti-choice conservatives: Feminist institutional networks in Mexico and Brazil (2000-2018). International Feminist Journal of Politics, 23(4), 600-624. https://doi.org/10.1080/14616742.2021.1954047

Zaremberg, G., Salas, K. y López Jara, M. D. (2019). "Buscando brújula: Política de transversalidad de género en el nivel municipal en México (2006-2018)”. En A. L. Rodríguez Gustá (Ed.), Marchas y contramarchas en las políticas locales de género. Dinámicas territoriales y ciudadanía de las mujeres en América Latina (pp. 229-257). CLACSO / Consejo Latinoamericano de Ciencias Sociales. 